\title{
Nonlinear Filtering for State Delayed Systems With Markovian Switching
}

\author{
Zidong Wang, Member, IEEE, James Lam, Senior Member, IEEE, and Xiaohui Liu
}

\begin{abstract}
This paper deals with the filtering problem for a general class of nonlinear time-delay systems with Markovian jumping parameters. The nonlinear time-delay stochastic systems may switch from one to the others according to the behavior of a Markov chain. The purpose of the problem addressed is to design a nonlinear full-order filter such that the dynamics of the estimation error is guaranteed to be stochastically exponentially stable in the mean square. Both filter analysis and synthesis problems are investigated. Sufficient conditions are established for the existence of the desired exponential filters, which are expressed in terms of the solutions to a set of linear matrix inequalities (LMIs). The explicit expression of the desired filters is also provided. A simulation example is given to illustrate the design procedures and performances of the proposed method.
\end{abstract}

Index Terms-Linear matrix inequalities, Markovian jump systems, nonlinear filtering, nonlinear systems, stochastic exponential stability, time-delay systems.

\section{INTRODUCTION}

$\mathbf{N}$ ONLINEAR filtering is one of the important issues in signal processing and has been an active research area over the past three decades. A recent overview on nonlinear filtering in the deterministic case can be found in [2]. For the stochastic case, the nonlinear filtering problem has been extensively studied. Due to the fact that the time evolution of the probability density of the state vector conditional on the measurements cannot be directly calculated in most cases, a lot of approximations have been developed in the literature, such as Gram-Charlier expansion, Edgeworth expansion, extended Kalman filters, weighted sum of Gaussian densities, generalized least-squares approximation, and statistically linearized filters; see, e.g., [4]. In particular, the nonlinear filtering problem was investigated in [15] through the concepts of observers for stochastic nonlinear systems, and an important stochastic stability approach to designing the observers with guaranteed convergence was developed. The results of [15] were then extended in [18] to more general stochastic nonlinear systems and measurement models, and excellent results were obtained that can ensure the exponential rate of convergence.

Manuscript received July 5, 2001; revised February 20, 2003. This work was supported in part by the University of Hong Kong under HKU CRCG Grant 10203795, the Engineering and Physical Sciences Research Council of U.K under Grants GR/S27658/01 and GR/R35018/01, and the Alexander von Humboldt Foundation of Germany. The associate editor coordinating the review of this paper and approving it for publication was Dr. Zhi-Quan (Tom) Luo.

Z. Wang and X. Liu are with the Department of Information Systems and Computing, Brunel University, Uxbridge, UB8 3PH, U.K. (e-mail: Zidong.Wang@brunel.ac.uk).

J. Lam is with the Department of Mechanical Engineering, The University of Hong Kong, Hong Kong.

Digital Object Identifier 10.1109/TSP.2003.815373
It is now well known that the delayed state is very often the cause for instability and poor performance of systems. In the past few years, we have seen an increasing interest in the control of linear systems with certain types of time-delays; see [12] for a survey. Concerning the robust and/or $H_{\infty}$ filtering of time-delay systems, see [11] and [17]. However, the nonlinear filtering problem for general time-delay stochastic systems has received very little attention. In [16], the nonlinear filtering problem was studied for uncertain time-delay stochastic systems where the nonlinearities were introduced in the form of additional nonlinear disturbances.

On the other hand, many physical systems are subject to frequent unpredictable structural changes, such as random failures, repairs of sudden environment disturbances, abrupt variation of the operating point on a nonlinear plant, etc. Markovian jump systems (MJSs), which comprise an important family of models subject to abrupt variations, are very often used to describe the above class of systems. Loosely speaking, a jump system is a hybrid one with state vector that has two components $x(t)$ and $r(t)$. The first one is in general referred to as the state, and the second one is regarded as the mode. In its operation, the jump system will switch from one mode to another in a random way, and the switching between the modes is governed by a Markov process with discrete and finite state space. In the past decade, the optimal regulator, controllability, observability, stability, and stabilization problems have been extensively studied for jump linear systems (JLSs); see, e.g., [6] and [10]. The stability analysis of a class of linear/nonlinear stochastic systems with Markovian switching has been addressed in [9], and a number of stability test criteria have been proposed. In addition, the filtering problem for JLSs has recently received initial attention; see [14] and references therein.

In practice, a nonlinear system with Markovian jumping parameters may be more reasonable to account for the nonlinearities and structural changes. To the best of the authors' knowledge, so far, there have been very few papers dealing with filter design problem for general nonlinear time-delay systems with or without Markovian jump parameters. This may be because the stochastic exponential stability analysis problem is quite involved for nonlinear time-delay jump systems. This situation encourages us to study the filtering problem for a class of nonlinear time-delay systems with Markovian switching.

This paper is concerned with the exponential filtering problem for nonlinear jump time-delay systems. Our aim is to design a nonlinear full-order filter such that the dynamics of the estimation error of each system mode is stochastically exponentially stable in the mean square. We show that both the filter analysis and the filter synthesis problems can be solved 
in terms of the solutions to a set of linear matrix inequalities (LMIs; see [1]). Note that over the past few years, LMIs have gained much attention for their computational tractability and usefulness in control engineering [1] as the so-called interior point method [3] has been proved to be numerically very efficient for solving the LMIs. Therefore, in our study, the powerful Matlab LMI toolbox [3] can be ideally employed to facilitate the filter design problem. We demonstrate the usefulness and applicability of the developed theory by means of a numerical simulation example.

Notation: The notations in this paper are quite standard. $\mathbb{R}^{n}$ and $\mathbb{R}^{n \times m}$ denote, respectively, the $n$ dimensional Euclidean space and the set of all $n \times m$ real matrices. The superscript " $T$ " denotes the transpose, and the notation $X \geq Y$ (respectively, $X>Y$ ), where $X$ and $Y$ are real symmetric matrices, means that $X-Y$ is positive semi-definite (respectively, positive definite). $I$ is the identity matrix with compatible dimension. We let $h>0$ and $C\left([-h, 0] ; \mathbb{R}^{n}\right)$ denote the family of continuous functions $\varphi$ from $[-h, 0]$ to $\mathbb{R}^{n}$ with the norm $\|\varphi\|=\sup _{-h \leq \theta \leq 0}|\varphi(\theta)|$, where $|\cdot|$ is the Euclidean norm in $\mathbb{R}^{n}$. If $A$ is a real matrix, denote by $\|A\|$ its operator norm, i.e., $\|A\|=\sup \{|A x|:|x|=1\}=\sqrt{\lambda_{\max }\left(A^{T} A\right)}$, where $\lambda_{\max }(\cdot)$ (respectively, $\left.\lambda_{\min }(\cdot)\right)$ means the largest (respectively, smallest) eigenvalue of $A . l_{2}[0, \infty]$ is the space of square integrable vector. Moreover, let $\left(\Omega, \mathcal{F},\left\{\mathcal{F}_{t}\right\}_{t>0}, P\right)$ be a complete probability space with a filtration $\left\{\mathcal{F}_{t}\right\}_{t>0}$ satisfying the usual conditions (i.e., the filtration contains all $P$-null sets and is right continuous). Denote by $L_{\mathcal{F}_{0}}^{p}\left([-h, 0] ; \mathbb{R}^{n}\right)$ the family of all $\mathcal{F}_{0}$-measurable $C\left([-h, 0] ; \mathbb{R}^{n}\right)$-valued random variables $\xi=\{\xi(\theta):-h \leq \theta \leq 0\}$ such that $\sup _{-h \leq \theta \leq 0} \mathbb{E}|\xi(\theta)|^{p}<\infty$, where $\mathbb{E}\{\cdot\}$ stands for the mathematical expectation operator with respect to the given probability measure $P$. The shorthand $\operatorname{diag}\left(M_{1}, M_{2}, \ldots, M_{N}\right)$ denotes a block diagonal matrix with diagonal blocks being the matrices $M_{1}, M_{2}, \ldots, M_{N}$. Sometimes, the arguments of a function will be omitted in the analysis when no confusion can arise.

\section{PROBLEM Formulation AND ASSUMPTIONS}

Let $\{r(t), t \geq 0\}$ be a right-continuous Markov process on the probability space which takes values in the finite space $\mathcal{S}=$ $\{1,2, \ldots, N\}$ with generator $\Pi=\left(\gamma_{i j}\right)(i, j \in \mathcal{S})$ given by

$$
\begin{aligned}
& P\{r(t+\Delta)=j \mid r(t)=i\} \\
&= \begin{cases}\gamma_{i j} \Delta+o(\Delta), & \text { if } i \neq j \\
1+\gamma_{i i} \Delta+o(\Delta), & \text { if } i=j\end{cases}
\end{aligned}
$$

where $\Delta>0$, and $\lim _{\Delta \rightarrow 0} O(\Delta) / \Delta=0, \gamma_{i j} \geq 0$ is the transition rate from $i$ to $j$ if $i \neq j$ and $\gamma_{i i}=-\sum_{j \neq i} \gamma_{i j}$.

Let us consider a nonlinear state delayed jump system in a fixed complete probability space $\left(\Omega, \mathcal{F},\left\{\mathcal{F}_{t}\right\}_{t>0}, P\right)$ described by

$$
\begin{aligned}
& \dot{x}(t)=f(x(t), r(t))+g(x(t-\tau), r(t)) \\
& x(t)=\varphi(t), \quad r(t)=r(0), \quad t \in[-\tau, 0] \\
& y(t)=h(x(t), r(t))
\end{aligned}
$$

where $x(t) \in \mathbb{R}^{n}$ is the state, $y(t) \in \mathbb{R}^{p}$ is the measurement output, and $f(\cdot, \cdot) \in \mathbb{R}^{n}, g(\cdot, \cdot) \in \mathbb{R}^{n}, h(\cdot, \cdot) \in \mathbb{R}^{p}$ are non- linear vector functions. $\tau$ denotes the state delay, and $\varphi(t)$ is a continuous vector valued initial function.

Assumption 1: For any fixed system mode $r(t)=i \in \mathcal{S}$, the nonlinear vector functions $f(\cdot, \cdot), g(\cdot, \cdot), h(\cdot, \cdot)$ are assumed to satisfy $f(0, i)=0, g(0, i)=0, h(0, i)=0$, and

$$
\begin{aligned}
& |f(x(t)+\sigma, i)-f(x(t), i)-A(i) \sigma| \leq a_{1}(i)|\sigma| \\
& \left|g(x(t-\tau)+\sigma, i)-g(x(t-\tau), i)-A_{d}(i) \sigma\right| \leq a_{2}(i)|\sigma| \\
& |h(x(t)+\sigma, i)-h(x(t), i)-C(i) \sigma| \leq a_{3}(i)|\sigma|
\end{aligned}
$$

where $A(i) \in \mathbb{R}^{n \times n}, A_{d}(i) \in \mathbb{R}^{n \times n}, C(i) \in \mathbb{R}^{p \times n}$ are known constant matrices, $\sigma \in \mathbb{R}^{n}$ is a vector, and $a_{1}(i), a_{2}(i)$, and $a_{3}(i)$ are known positive constants.

Remark 1: The nonlinear descriptions (4)-(6) (see [18]) reflect the "distance" between the originally nonlinear model (1)-(3) and the "nominal" linear model, whose system parameters are $A(i), A_{d}(i), C(i)$. Such nonlinear descriptions are actually similar to the Lipschitz conditions on the nonlinear functions $f(\cdot, \cdot), g(\cdot, \cdot), h(\cdot, \cdot)$. In applications, the linearization technique may be exploited to quantify the maximum possible derivations from the nominal model. One of the future research topics is to describe the nonlinearities in a more general way.

Throughout this paper, we will employ the full-order nonlinear filter that is of the following structure:

$$
\begin{aligned}
\dot{\hat{x}}(t)=f(\hat{x}(t), r(t))+g & (\hat{x}(t-\tau), r(t)) \\
& +K(r(t))[y(t)-h(\hat{x}(t), r(t))]
\end{aligned}
$$

where $\hat{x}$ is the state estimate, and the constant gains $K(r(t))$ are the filter parameters to be designed.

Notice that the Markov process $\{r(t), t \geq 0\}$ takes values in the finite space $\mathcal{S}=\{1,2, \ldots, N\}$. For notational convenience, we write

$$
\begin{aligned}
A(i) & :=A_{i}, \quad A_{d}(i):=A_{d i}, \quad C(i):=C_{i} \\
a_{11}(i) & :=a_{11 i}, \quad a_{22}(i):=a_{22 i}, \quad a_{33}(i):=a_{33 i} .
\end{aligned}
$$

Let the error state be $e(t)=x(t)-\hat{x}(t)$. It then follows from (1)-(3) and (7) that

$$
\begin{aligned}
\dot{e}(t)= & f(x(t), r(t))-f(\hat{x}(t), r(t)) \\
& +g(x(t-\tau), r(t))-g(\hat{x}(t-\tau), r(t)) \\
& -K(r(t))[h(x(t), r(t))-h(\hat{x}(t), r(t))] .
\end{aligned}
$$

Now, we will work on the system mode $r(t)=i, \forall i \in \mathcal{S}$. To continue, we introduce the following definitions:

$$
\begin{aligned}
l_{i}(t):= & f(x(t), i)-f(\hat{x}(t), i)-A(i) e(t) \\
m_{i}(t-\tau):= & g(x(t-\tau), i)-g(\hat{x}(t-\tau), i) \\
& -A_{d}(i) e(t-\tau) \\
n_{i}(t):= & h(x(t), i)-h(\hat{x}(t), i)-C(i) e(t) .
\end{aligned}
$$

Then, we can obtain from (8)-(13) that

$$
\begin{aligned}
\dot{e}(t)=\left(A_{i}-K_{i} C_{i}\right) e(t)+A_{d i} e(t-\tau)+l_{i}(t) \\
+m_{i}(t-\tau)-K_{i} n_{i}(t) .
\end{aligned}
$$


Now, let $e(t ; \xi)$ denote the state trajectory from the initial data $e(\theta)=\xi(\theta)$ on $-\tau \leq \theta \leq 0$ in $L_{\mathcal{F}_{0}}^{2}\left([-\tau, 0] ; \mathbb{R}^{n}\right)$. It is clear from our Assumption 1 that the system (14) admits a trivial solution $e(t ; 0) \equiv 0$ corresponding to the initial data $\xi=0$.

Definition 1: Consider the error dynamic system (14). For every $\xi \in L_{\mathcal{F}_{0}}^{2}\left([-\tau, 0] ; \mathbb{R}^{n}\right)$, we have the following.

- The trivial solution is asymptotically stable in the mean square if

$$
\lim _{t \rightarrow \infty} \mathbb{E}|e(t ; \xi)|^{2}=0 .
$$

- The trivial solution is exponentially stable in the mean square if there exist constants $\alpha>0$ and $\beta>0$ such that

$$
\mathbb{E}|e(t ; \xi)|^{2} \leq \alpha e^{-\beta t} \sup _{-\tau \leq \theta \leq 0} \mathbb{E}|\xi(\theta)|^{2}
$$

Definition 2: The filter (7) is said to be exponential (respectively, asymptotic) if, for every $\xi \in L_{\mathcal{F}_{0}}^{2}\left([-\tau, 0] ; \mathbb{R}^{n}\right)$, the system (14) is exponentially stable (respectively, asymptotically stable) in the mean square.

The primary objective of this paper is to provide a practical design procedure for an exponential filter of the nonlinear timedelay system (1)-(3). In other words, we will design the filter parameter $K_{i}$ such that the dynamics of the estimation error (i.e., the solution of the system (14)) is guaranteed to be stochastically exponentially stable.

\section{MAin Results AND PROOFS}

In this section, we will obtain a solution to the filter design problem formulated in the previous section by using a linear matrix inequality approach.

We first recall several lemmas that will be needed in the proof of our main results.

Lemma 1 (Schur Complement): Given constant matrices $\Omega_{1}, \Omega_{2}, \Omega_{3}$ where $\Omega_{1}=\Omega_{1}^{T}$ and $0<\Omega_{2}=\Omega_{2}^{T}$, then $\Omega_{1}+\Omega_{3}^{T} \Omega_{2}^{-1} \Omega_{3}<0$ if and only if

$$
\left[\begin{array}{cc}
\Omega_{1} & \Omega_{3}^{T} \\
\Omega_{3} & -\Omega_{2}
\end{array}\right]<0
$$

or

$$
\left[\begin{array}{cc}
-\Omega_{2} & \Omega_{3} \\
\Omega_{3}^{T} & \Omega_{1}
\end{array}\right]<0
$$

Lemma 2: Let $x \in \mathbb{R}^{n}, y \in \mathbb{R}^{n}$ and $\varepsilon>0$. Then, we have

$$
x^{T} y+y^{T} x \leq \varepsilon x^{T} x+\varepsilon^{-1} y^{T} y .
$$

Proof: The proof follows from the inequality

$$
\left(\varepsilon^{1 / 2} x-\varepsilon^{-1 / 2} y\right)^{T}\left(\varepsilon^{1 / 2} x-\varepsilon^{-1 / 2} y\right) \geq 0
$$

immediately.

Lemma 3 [5]: Let $X \in \mathbb{R}^{m_{1} \times n_{1}}, Y \in \mathbb{R}^{m_{1} \times p_{1}}\left(m_{1} \leq p_{1}\right)$. There exists a matrix $U \in \mathbb{R}^{n_{1} \times p_{1}}$ that simultaneously satisfies

$$
Y=X U, \quad U U^{T}=I
$$

if and only if

$$
X X^{T}=Y Y^{T} .
$$

The following theorem, which acts as a main key for solving the addressed nonlinear filtering problem, shows that the exponential stability of a given filter for the nonlinear time-delay stochastic system (1)-(3) can be guaranteed if positive definite solutions to a set of modified algebraic Riccati-like matrix inequalities (quadratic matrix inequalities) are known to exist.

Theorem 1: Let the filter parameters $K_{i}$ be given. If there exists a sequence of positive scalars $\left\{\varepsilon_{1 i}, \varepsilon_{2 i}, \varepsilon_{3 i}, \varepsilon_{4 i}, i \in \mathcal{S}\right\}$ such that the following matrix inequalities

$$
\begin{aligned}
& \sum_{j=1}^{N} \gamma_{i j}\left(\varepsilon_{1 j}^{-1} A_{d j}^{T} A_{d j}+\varepsilon_{3 j}^{-1} a_{22 j}^{2} I\right) \leq 0 \\
& \left(A_{i}-K_{i} C_{i}\right)^{T} P_{i}+P_{i}\left(A_{i}-K_{i} C_{i}\right)+\sum_{j=1}^{N} \gamma_{i j} P_{j} \\
& \quad+P_{i}\left[\left(\varepsilon_{1 i}+\varepsilon_{2 i}+\varepsilon_{3 i}\right) I+\varepsilon_{4 i} K_{i} K_{i}^{T}\right] P_{i} \\
& \quad+\left(\varepsilon_{2 i}^{-1} a_{11 i}^{2}+\varepsilon_{4 i}^{-1} a_{33 i}^{2}\right) I+Q_{i}<0
\end{aligned}
$$

where

$$
Q_{i}:=\varepsilon_{1 i}^{-1} A_{d i}^{T} A_{d i}+\varepsilon_{3 i}^{-1} a_{22 i}^{2} I
$$

have positive definite solutions $P_{i}>0$, then system (14) is exponentially stable in the mean square.

Proof: Fix $\xi \in L_{\mathcal{F}_{0}}^{2}\left([-\tau, 0] ; \mathbb{R}^{n}\right)$ arbitrarily, and write $e(t ; \xi)=e(t)$. For $(e(t), t) \in \mathbb{R}^{n} \times \mathbb{R}_{+}$, we define the stochastic Lyapunov functional $V(\cdot): \mathbb{R}^{n} \times \mathbb{R}_{+} \times \mathcal{S} \rightarrow \mathbb{R}_{+}$as

$$
\begin{aligned}
V(e(t), r(t)=i) & :=V(e(t), t, i) \\
& =e^{T}(t) P_{i} e(t)+\int_{t-\tau}^{t} e^{T}(s) Q_{i} e(s) d s
\end{aligned}
$$

where $P_{i}$ is the positive definite solution to the matrix inequality (18), and $Q_{i}>0$ is defined in (19).

The weak infinitesimal operator $\mathcal{A}$ (see [6]) of the stochastic process $\{r(t), e(t)\}(t \geq 0)$ is given by

$$
\begin{aligned}
\mathcal{A} V( & (t), r(t)) \\
= & \lim _{\Delta \rightarrow 0} \frac{1}{\Delta}[\mathbb{E}\{V(x(t+\Delta), r(t+\Delta)) \mid x(t), r(t)=i\} \\
& -V(x(t), r(t)=i)] \\
= & e^{T}(t)\left[\left(A_{i}-K_{i} C_{i}\right)^{T} P_{i}+P_{i}\left(A_{i}-K_{i} C_{i}\right)\right. \\
& \left.+\sum_{j=1}^{N} \gamma_{i j} P_{j}+Q_{i}\right] e(t) \\
& +e^{T}(t) P_{i} A_{d i} e(t-\tau)+e^{T}(t-\tau) A_{d i}^{T} P_{i} e(t) \\
& +e^{T}(t) P_{i}\left[l_{i}(t)+m_{i}(t-\tau)-K_{i} n_{i}(t)\right] \\
& +\left[l_{i}(t)+m_{i}(t-\tau)-K_{i} n_{i}(t)\right]^{T} P_{i} e(t) \\
& -e^{T}(t-\tau) Q_{i} e(t-\tau) \\
& +\sum_{j=1}^{N} \gamma_{i j} \int_{t-\tau}^{t} e^{T}(s) Q_{j} e(s) d s .
\end{aligned}
$$


It is obvious from (17) and (19) that

$$
\sum_{j=1}^{N} \gamma_{i j} \int_{t-\tau}^{t} e^{T}(s) Q_{j} e(s) d s \leq 0 .
$$

Let $\varepsilon_{1 i}, \varepsilon_{2 i}, \varepsilon_{3 i}, \varepsilon_{4 i}$ be positive scalars. It then follows from Lemma 2 that

$$
\begin{aligned}
& e^{T}(t) P_{i} A_{d i} e(t-\tau)+e^{T}(t-\tau) A_{d i}^{T} P_{i} e(t) \\
& \quad \leq \varepsilon_{1 i} e^{T}(t) P_{i}^{2} e(t)+\varepsilon_{1 i}^{-1} e^{T}(t-\tau) A_{d i}^{T} A_{d i} e(t-\tau) .
\end{aligned}
$$

In addition, it results from Assumption 1, the definitions (8), (9), and (11)-(13), that

$$
\begin{aligned}
l_{i}^{T}(t) l_{i}(t) & \leq a_{11 i}^{2}|e(t)|^{2}=a_{11 i}^{2} e^{T}(t) e(t) \\
m_{i}^{T}(t-\tau) m_{i}(t-\tau) & \leq a_{22 i}^{2} e^{T}(t-\tau) e(t-\tau) \\
n_{i}^{T}(t) n_{i}(t) & \leq a_{33 i}^{2}|e(t)|^{2}=a_{33 i}^{2} e^{T}(t) e(t) .
\end{aligned}
$$

Considering (24)-(26), we can obtain from Lemma 2 that

$$
\begin{aligned}
& e^{T}(t) P_{i} l_{i}(t)+l_{i}^{T}(t) P_{i} e(t) \\
& \quad \leq e^{T}(t)\left(\varepsilon_{2 i} P_{i}^{2}+\varepsilon_{2 i}^{-1} a_{11 i}^{2} I\right) e(t) \\
& e^{T}(t) P_{i} m_{i}(t-\tau)+m_{i}^{T}(t-\tau) P_{i} e(t) \\
& \quad \leq \varepsilon_{3 i} e^{T}(t) P_{i}^{2} e(t)+\varepsilon_{3 i}^{-1} a_{22 i}^{2} e^{T}(t-\tau) e(t-\tau) \\
& \quad-e^{T}(t) P_{i} K_{i} n_{i}(t)-n_{i}^{T}(t) K_{i}^{T} P_{i} e(t) \\
& \quad \leq \varepsilon_{4 i} e^{T}(t)\left(P_{i} K_{i} K_{i}^{T} P_{i}\right) e(t)+\varepsilon_{4 i}^{-1} a_{33 i}^{2} e^{T}(t) e(t) .
\end{aligned}
$$

For simplicity, we denote

$$
\begin{aligned}
\Pi_{i}:= & \left(A_{i}-K_{i} C_{i}\right)^{T} P_{i}+P_{i}\left(A_{i}-K_{i} C_{i}\right)+\sum_{j=1}^{N} \gamma_{i j} P_{j} \\
& +P_{i}\left[\left(\varepsilon_{1 i}+\varepsilon_{2 i}+\varepsilon_{3 i}\right) I+\varepsilon_{4 i} K_{i} K_{i}^{T}\right] P_{i} \\
& +\left(\varepsilon_{2 i}^{-1} a_{11 i}^{2}+\varepsilon_{4 i}^{-1} a_{33 i}^{2}\right) I \\
& +\varepsilon_{1 i}^{-1} A_{d i}^{T} A_{d i}+\varepsilon_{3 i}^{-1} a_{22 i}^{2} I
\end{aligned}
$$

then (18) and (19) result in that $\Pi_{i}<0$.

Substituting (19), (22), (23), and (27)-(29) into (21) yields

$$
\mathcal{A} V(e(t), i) \leq e^{T}(t) \Pi_{i} e(t) \leq-\lambda_{\min }\left(-\Pi_{i}\right) e^{T}(t) e(t)
$$

To show the expected exponential stability (in the mean square) of the system (14), we need to perform some standard manipulations on (31) by utilizing the technique developed in [7] and [8]. The details are along the similar line of the proof of [8, Th. 2.1] and are thus omitted here. We just mention that for the exponential stability of (14), the required constant $\beta>0$ in (16) is the unique root of the equation

$$
\lambda_{\min }\left(-\Pi_{i}\right)-\beta \lambda_{\max }\left(P_{i}\right)-\beta \tau \lambda_{\max }\left(Q_{i}\right) e^{\beta \tau}=0
$$

and the required constant $\alpha>0$ can be determined by $\alpha:=\lambda_{\min }^{-1}\left(P_{i}\right)\left[\lambda_{\max }\left(P_{i}\right)+\tau \lambda_{\max }\left(Q_{i}\right)\left(1+\tau e^{\beta \tau}\right)\right]$. This completes the proof of Theorem 1.

Remark 2: In Theorem 1, we establish the analysis results for the exponential stability of the nonlinear time-delay jump systems. That is, for a given filter structure, we derive the sufficient conditions under which the estimation error dynamics are stochastically exponentially stable in the mean square. We arrive at the conclusion that the solvability of the addressed particular nonlinear filtering problem is closely related to the solutions to a set of quadratic matrix inequalities.

The following corollary reveals that for the nonlinear timedelay jump system (14), the exponential stability in the mean square also implies the almost-surely exponential stability. The proof can be found in [7].

Corollary 1: Under the same conditions as in Theorem 1, the nonlinear time-delay system (14) is almost-surely exponentially stable in the mean square. That is

$$
\lim _{t \rightarrow \infty} \sup \frac{1}{t} \log |e(t ; \xi)| \leq-\frac{\beta}{2}
$$

almost surely holds for all $\xi \in L_{\mathcal{F}_{0}}^{2}\left([-\tau, 0] ; \mathbb{R}^{n}\right)$, where $\beta>0$ is the the unique root of the (32).

Having obtained the analysis results in Theorem 1, we are now ready to tackle the corresponding synthesis problem. That is, we need to derive the explicit expression of expected filter gains and propose a practical design procedure. It should be pointed out that in most literature concerning nonlinear filtering, the solution to the nonlinear filtering problem has not been given as an explicit representation.

For presentation convenience, we further define

$$
\begin{aligned}
& \Gamma_{i}:=A_{i}^{T} P_{i}+P_{i} A_{i}+\sum_{j=1}^{N} \gamma_{i j} P_{j}+\left(\varepsilon_{1 i}+\varepsilon_{2 i}+\varepsilon_{3 i}\right) P_{i}^{2} \\
& +\left(\varepsilon_{2 i}^{-1} a_{11 i}^{2}+\varepsilon_{4 i}^{-1} a_{33 i}^{2}\right) I+Q_{i} \\
& \Xi_{i}:=A_{i}^{T} P_{i}+P_{i} A_{i}+\sum_{j=1}^{N} \gamma_{i j} P_{j}+\left(\varepsilon_{1 i}+\varepsilon_{2 i}+\varepsilon_{3 i}\right) P_{i}^{2} \\
& +\varepsilon_{1 i}^{-1} A_{d i}^{T} A_{d i}+\left(\varepsilon_{2 i}^{-1} a_{11 i}^{2}+\varepsilon_{3 i}^{-1} a_{22 i}^{2}+\varepsilon_{4 i}^{-1} a_{33 i}^{2}\right) I \\
& -\varepsilon_{4 i}^{-1} C_{i}^{T} C_{i} \\
& \Theta_{i}:=\left[\begin{array}{llllll}
P_{i} & \mu_{1 i} A_{d i}^{T} & P_{i} & \mu_{2 i} a_{11 i} I & P_{i} & \mu_{3 i} a_{22 i} I
\end{array}\right]
\end{aligned}
$$

where $Q_{i}$ is defined in (19).

In principle, our task now consists of two parts. One is to find the necessary and sufficient conditions for the existence of filter gains $K_{i}$ such that there exist positive definite matrices $P_{i}$ satisfying (18), and the other one is to express all expected filter gains in terms of the positive definite solutions $P_{i}$ and, if any, some other free parameters. The following theorem accomplishes the above specified task.

Theorem 2: There exist a sequence of positive scalars $\left\{\varepsilon_{1 i}, \varepsilon_{2 i}, \varepsilon_{3 i}, \varepsilon_{4 i}, i \in \mathcal{S}\right\}$ and positive definite matrices $P_{i}$ such that the matrix inequalities (18) (for $i \in \mathcal{S}$ ) have solutions $K_{i}$ if and only if one of the following two assertions holds.

1) There exist a sequence of positive scalars $\left\{\varepsilon_{1 i}, \ldots\right.$, $\left.\varepsilon_{4 i}, i \in \mathcal{S}\right\}$ and positive definite matrices $P_{i}$ such that $\Xi_{i}<0$, where $\Xi$ is defined in (34).

2) There exist a sequence of positive scalars $\left\{\mu_{1 i}, \ldots\right.$, $\left.\mu_{4 i}, i \in \mathcal{S}\right\}$ and positive definite matrices $P_{i}$ such that the following set of linear matrix inequalities

$$
\left[\begin{array}{cc}
\Upsilon_{i} & \Theta_{i} \\
\Theta_{i}^{T} & -\operatorname{diag}\left\{\mu_{1 i} I, \mu_{1 i} I, \mu_{2 i} I, \mu_{2 i} I, \mu_{3 i} I, \mu_{3 i} I\right\}
\end{array}\right]<0
$$


hold, where

$$
\Upsilon_{i}:=A_{i}^{T} P_{i}+P_{i} A_{i}+\sum_{j=1}^{N} \gamma_{i j} P_{j}+\mu_{4 i}\left(a_{33 i}^{2} I-C_{i}^{T} C_{i}\right)
$$

Furthermore, if (36) is true for positive scalars $\mu_{1 i}, \mu_{2 i}, \mu_{3 i}, \mu_{4 i}$ and positive definite matrices $P_{i}$, all matrices $K_{i}$ meeting the matrix inequalities (18) can then be parameterized by

$$
K_{i}=\mu_{4 i} P_{i}^{-1} C_{i}^{T}+\mu_{4 i}^{1 / 2} P_{i}^{-1} \Lambda_{i} U_{i}
$$

where $\Lambda_{i} \in \mathbb{R}^{n \times p}$ is any matrix satisfying

$$
\Lambda_{i} \Lambda_{i}^{T}<-\Xi_{i}
$$

for $\varepsilon_{k i}=\mu_{k i}^{-1}(k=1,2,3,4)$, and $U_{i} \in \mathbb{R}^{p \times p}$ is an arbitrary orthogonal matrix (i.e., $U_{i} U_{i}^{T}=I$ ).

Proof: It is straightforward to rearrange the matrix inequality (18) as

$$
-C_{i}^{T} K_{i}^{T} P_{i}-P_{i} K_{i} C_{i}+\varepsilon_{4 i} P_{i} K_{i} K_{i}^{T} P_{i}+\Gamma_{i}<0
$$

where $\Gamma_{i}$ is defined in (33), or

$$
\begin{aligned}
{\left[\varepsilon_{4 i}^{1 / 2} P_{i} K_{i}-\varepsilon_{4 i}^{-1 / 2} C_{i}^{T}\right]\left[\varepsilon_{4 i}^{1 / 2} P_{i} K_{i}\right.} & \left.-\varepsilon_{4 i}^{-1 / 2} C_{i}^{T}\right]^{T} \\
& <\varepsilon_{4 i}^{-1} C_{i}^{T} C_{i}-\Gamma_{i} .
\end{aligned}
$$

It is apparent that there exist filter gain matrices $K_{i}$ such that the inequalities (18) (or equivalently, (41) for $i \in \mathcal{S}$ ) hold for some positive scalars $\varepsilon_{1 i}, \varepsilon_{2 i}, \varepsilon_{3 i}, \varepsilon_{4 i}$ and positive definite matrix $P_{i}$ if and only if the right-hand side of (41) is positive definite. That is

$$
\begin{aligned}
& A_{i}^{T} P_{i}+P_{i} A_{i}+\sum_{j=1}^{N} \gamma_{i j} P_{j}+\left(\varepsilon_{1 i}+\varepsilon_{2 i}+\varepsilon_{3 i}\right) P_{i}^{2} \\
& \quad+\varepsilon_{1 i}^{-1} A_{d i}^{T} A_{d i}+\left(\varepsilon_{2 i}^{-1} a_{11 i}^{2}+\varepsilon_{3 i}^{-1} a_{22 i}^{2}\right. \\
& \left.\quad+\varepsilon_{4 i}^{-1} a_{33 i}^{2}\right) I-\varepsilon_{4 i}^{-1} C_{i}^{T} C_{i}<0
\end{aligned}
$$

or $\Xi_{i}<0$ holds.

Notice that (42) is neither linear on $P_{i}$ nor linear on $\varepsilon_{1 i}, \varepsilon_{2 i}, \varepsilon_{3 i}, \varepsilon_{4 i}$. Our next goal is to convert (42) into an LMI so that the powerful Matlab LMI Toolbox can be applied. To do this, we continue to rewrite (42) as

$$
\Upsilon_{i}+\Omega_{i} \Omega_{i}^{T}<0
$$

where $\Upsilon_{i}$ is defined in (37) (let $\mu_{4 i}:=\varepsilon_{4 i}^{-1}$ ), and

$$
\Omega_{i}:=\left[\begin{array}{ll}
\Omega_{1 i} & \Omega_{2 i}
\end{array}\right]
$$

where

$$
\begin{aligned}
& \Omega_{1 i}=\left[\begin{array}{lll}
\varepsilon_{1 i}^{1 / 2} P_{i} & \varepsilon_{1 i}^{-1 / 2} A_{d i}^{T} & \varepsilon_{2 i}^{1 / 2} P_{i}
\end{array}\right] \\
& \Omega_{2 i}=\left[\begin{array}{lll}
\varepsilon_{2 i}^{-1 / 2} a_{11 i} I & \varepsilon_{3 i}^{1 / 2} P_{i} & \varepsilon_{3 i}^{-1 / 2} a_{22 i} I
\end{array}\right] .
\end{aligned}
$$

It follows from the Schur complement lemma (Lemma 1) that (43) holds if and only if the following inequality holds:

$$
\left[\begin{array}{cc}
\Upsilon_{i} & \Omega_{i} \\
\Omega_{i}^{T} & -I
\end{array}\right]<0
$$

Let

$$
\mu_{k i}:=\varepsilon_{k i}^{-1}, \quad k=1,2,3,4 .
$$

Pre- and post-multiplying the inequality (45) by

$$
\operatorname{diag}\left\{I, \varepsilon_{1 i}^{-1 / 2} I, \varepsilon_{1 i}^{-1 / 2} I, \varepsilon_{2 i}^{-1 / 2} I, \varepsilon_{2 i}^{-1 / 2} I, \varepsilon_{3 i}^{-1 / 2} I, \varepsilon_{3 i}^{-1 / 2} I\right\}
$$

yield (36). This proves the first part of this theorem.

Suppose now that (36) is true. Note that the dimension of the filter gain $K_{i}$ is $n \times p$ and $p \leq n$. From (41) and the definition of $\Lambda_{i} \in \mathbb{R}^{n \times p}$ in (39), we have

$$
\left[\varepsilon_{4 i}^{1 / 2} P_{i} K_{i}-\varepsilon_{4 i}^{-1 / 2} C_{i}^{T}\right]\left[\varepsilon_{4 i}^{1 / 2} P_{i} K_{i}-\varepsilon_{4 i}^{-1 / 2} C_{i}^{T}\right]^{T}=\Lambda_{i} \Lambda_{i}^{T} .
$$

It then follows from Lemma 3 that (47) holds if and only if

$$
\varepsilon_{4 i}^{1 / 2} P_{i} K_{i}-\varepsilon_{4 i}^{-1 / 2} C_{i}^{T}=\Lambda_{i} U_{i}
$$

where $U_{i} \in \mathbb{R}^{p \times p}$ is an arbitrary orthogonal matrix. Therefore, (38) follows from (48) immediately, and the proof of this theorem is complete.

Noticing that (17) is the same as the LMI

$$
\sum_{j=1}^{N} \gamma_{i j}\left(\mu_{1 j} A_{d j}^{T} A_{d j}+\mu_{3 j} a_{22 j}^{2} I\right) \leq 0
$$

we summarize our main results as follows, which are easily derived from Theorems 1 and 2 .

Theorem 3: Consider the nonlinear jump state delayed system (1)-(3) and the corresponding nonlinear filter (7). If there exist a sequence of positive scalars $\left\{\mu_{1 i}, \mu_{2 i}, \mu_{3 i}, \mu_{4 i}, i \in \mathcal{S}\right\}$ and positive definite matrices $P_{i}(i \in \mathcal{S})$ such that the LMIs (36) and (49) hold, then the filter (7) with its parameter given in (38) will be such that the dynamics of the estimation error (i.e., the solution of the error-state system (14)) are stochastically exponentially stable in the mean square.

Remark 3: The solution to the addressed filter design problem for nonlinear jump time-delay systems is given in Theorem 3. Note that the design procedure of the filter parameters depends solely on the feasibility of the LMIs (36) and (49) that are linear on the scalar variables $\mu_{1 i}>0, \mu_{2 i}>0, \mu_{3 i}>0, \mu_{4 i}>0$ and the matrix variable $P_{i}>0$. Fortunately, with the recently developed Matlab LMI Toolbox [3], we can check the solvability of the LMIs (36) and (49) readily and reliably. This makes our proposed design approach very practical.

Remark 4: We can see that if the set of desired filter gains is not empty, it is often very large. We may utilize the freedom (such as the choices of matrices $\Lambda_{i}$ and $U_{i}$ ) in the filter design to improve other system properties. One of the future research topics is to exploit such remaining freedom to achieve the specified reliable constraint on the filtering process. It would also be interesting to study the case when the nonlinear function $g(x(t-\tau), r(t))$ is regarded as an unknown perturbation. Finally, we point out that it is not difficult to obtain parallel results for both the multidelay case and for the case where there are bounded nonlinearities and uncertain disturbances. The reason why we discuss the relatively simple system (1)-(3) associated with (4)-(6) is to make our theory more understandable and to avoid unnecessarily complicated notations. 


\section{NUMERICAL EXAMPLE}

In this section, a numerical example is presented to illustrate the usefulness and flexibility of the developed theory.

The nonlinear time-delay jump system under consideration is assumed to have two modes. The Markov process that governs the mode switching has generator $\Pi=\left(\gamma_{i j}\right)(i, j=1,2)$, where

$$
\gamma_{11}=-3, \quad \gamma_{12}=3, \quad \gamma_{21}=0.4, \quad \gamma_{22}=-0.4 .
$$

Let the system mode 1 be given by

$$
\begin{aligned}
\dot{x}_{1}(t)= & -2.8 x_{1}(t)+0.1 x_{2}(t) \\
& +0.2 \sin \left(x_{1}(t)+x_{2}(t)\right) \\
& +0.05 x_{1}(t-0.1)-0.02 x_{2}(t-0.1) \\
\dot{x}_{2}(t)= & 0.4 x_{1}(t)-3.2 x_{2}(t)+0.3 \cos x_{2}(t) \\
& +0.01 x_{1}(t-0.1)-0.03 x_{2}(t-0.1) \\
y_{1}(t)= & 0.95 x_{1}(t)+0.2 \sin x_{2}(t), \\
y_{2}(t)= & 0.95 x_{2}(t)+0.1 \cos x_{1}(t)
\end{aligned}
$$

and the system mode 2 be described by

$$
\begin{aligned}
\dot{x}_{1}(t)= & -1.9 x_{1}(t)-0.2 x_{2}(t) \\
& +0.3 \sin \left(x_{1}(t)-2 x_{2}(t)\right) \\
& +0.08 x_{1}(t-0.1)+0.06 x_{2}(t-0.1) \\
\dot{x}_{2}(t)= & 0.3 x_{1}(t)-4.5 x_{2}(t)+0.4 \cos x_{2}(t) \\
& +0.02 x_{1}(t-0.1)+0.05 x_{2}(t-0.1) \\
y_{1}(t)= & 0.98 x_{1}(t)+0.1 \sin x_{2}(t) \\
y_{2}(t)= & 0.98 x_{2}(t)+0.2 \cos x_{1}(t) .
\end{aligned}
$$

Considering the system (1)-(3) with the constraints (4)-(6), we can obtain the system data as follows:

$$
\begin{aligned}
A_{1} & =\left[\begin{array}{cc}
-2.8 & 0.1 \\
0.4 & -3.2
\end{array}\right], \quad A_{2}=\left[\begin{array}{cc}
-1.9 & -0.2 \\
0.3 & -4.5
\end{array}\right] \\
A_{d 1} & =\left[\begin{array}{ll}
0.05 & -0.02 \\
0.01 & -0.03
\end{array}\right], \quad A_{d 2}=\left[\begin{array}{cc}
0.08 & 0.06 \\
0.02 & 0.05
\end{array}\right] \\
C_{1} & =0.95 I_{2}, \quad C_{2}=0.98 I_{2}, \quad \Pi=\left[\begin{array}{cc}
-3 & 3 \\
0.4 & -0.4
\end{array}\right] \\
a_{11} & =0.37, \quad a_{21}=0, \quad a_{31}=0.23, \quad a_{12}=0.50 \\
a_{22} & =0, \quad a_{32}=0.23, \quad \tau=0.1, \quad \varphi(t)=0.1 .
\end{aligned}
$$

Solving the LMIs (36) and (49) $(i=1,2)$ by using the LMI toolbox [3], we obtain that

$$
\begin{aligned}
\mu_{11} & =2.4876, \quad \mu_{12}=1.5076 \\
\mu_{21} & =1.5721, \quad \mu_{22}=2.0567 \\
\mu_{31} & =1.0275, \quad \mu_{32}=1.4356 \\
\mu_{41} & =2.8835, \quad \mu_{42}=2.5592 \\
P_{1} & =\left[\begin{array}{cc}
3.2020 & -0.3010 \\
-0.3010 & 2.3636
\end{array}\right] \\
P_{2} & =\left[\begin{array}{cc}
3.1106 & -0.0388 \\
-0.0388 & 2.7748
\end{array}\right] .
\end{aligned}
$$

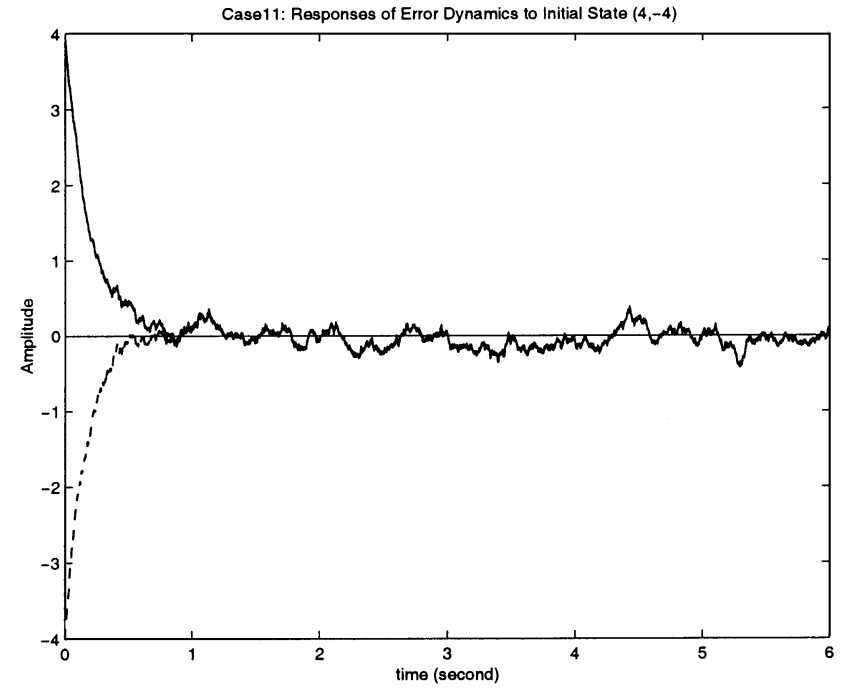

Fig. 1. $e_{1}$ (solid), $e_{2}$ (dash)

We are now ready to compute the desired filter parameters $K_{i}(i=1,2)$. To illustrate the design flexibility, we will make use of the freedom in selecting the parameters $\Lambda_{i}$ and $U_{i}$. We first consider the system mode 1 .

Case 11: In this case, we choose $\Lambda_{1}$ (meeting $\Lambda_{i} \Lambda_{i}^{T}<-\Xi_{i}$ ) and the orthogonal matrix $U_{1}$ as $\Lambda_{1}=2 I_{2}, U_{1}=I_{2}$. It then follows from (38) that

$$
K_{1}=\left[\begin{array}{ll}
1.9393 & 0.2470 \\
0.2470 & 2.6273
\end{array}\right] \text {. }
$$

Denote the error states $e_{i}=x_{i}-\hat{x}_{i}(i=1,2)$. The responses of the error dynamics to initial conditions are shown in Fig. 1. The simulation result implies that the desired goal is achieved.

Case 12: In this case, we select $\Lambda_{1}$ (meeting $\Lambda_{i} \Lambda_{i}^{T}<-\Xi_{i}$ ), which is the orthogonal matrix $U_{1}$ and therefore obtain the filter gain $K_{1}$, respectively, as follows:

$$
\begin{aligned}
\Lambda_{1} & =1.8 I_{2}, \quad U_{1}=\operatorname{diag}\{1,-1\} \\
K_{1} & =\left[\begin{array}{ll}
1.8320 & -0.0128 \\
0.2333 & -0.1358
\end{array}\right] .
\end{aligned}
$$

The responses of the error dynamics to initial conditions are shown in Fig. 2.

Next, let us consider the system mode 2 .

Case 21: In this case, we set $\Lambda_{2}$ as $\Lambda_{2}=1.2 I_{2}$ and the orthogonal matrix $U_{2}$ as $U_{2}=I_{2}$. The filter gain is then calculated as

$$
K_{2}=\left[\begin{array}{ll}
1.4237 & 0.0199 \\
0.0199 & 1.5960
\end{array}\right]
$$

and the simulation result is given in Fig. 3, which indicates that our expected performance is guaranteed.

Case 22: We now let $\Lambda_{2}$ be $\Lambda_{2}=0.9 I_{2}$ and the orthogonal matrix $U_{2}$ be $U_{2}=\operatorname{diag}\{-1,1\}$. Then, we obtain

$$
K_{2}=\left[\begin{array}{ll}
0.3828 & 0.0487 \\
0.0487 & 0.5186
\end{array}\right] \text {. }
$$

and give the simulation result in Fig. 4. 


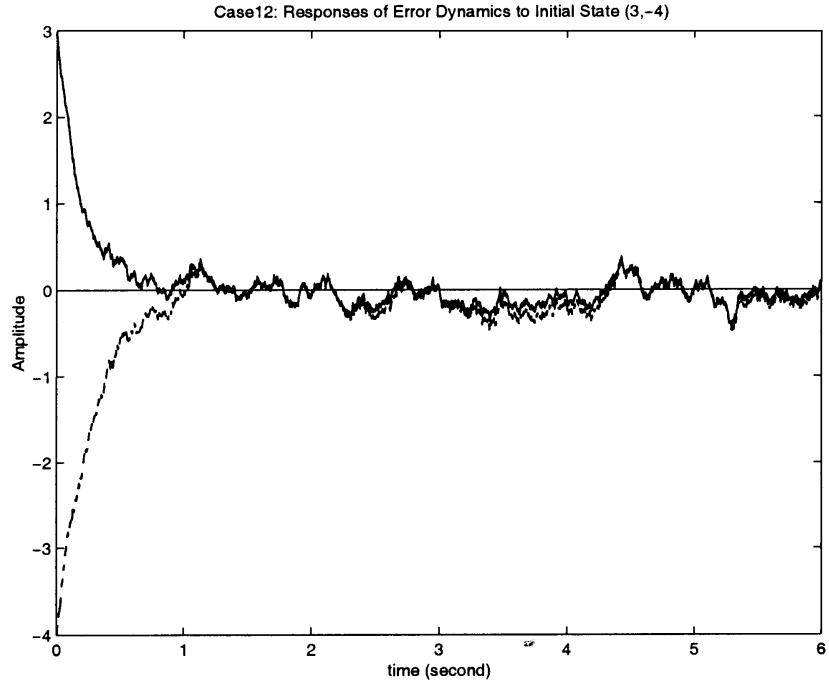

Fig. 2. $e_{1}$ (solid), $e_{2}$ (dash).

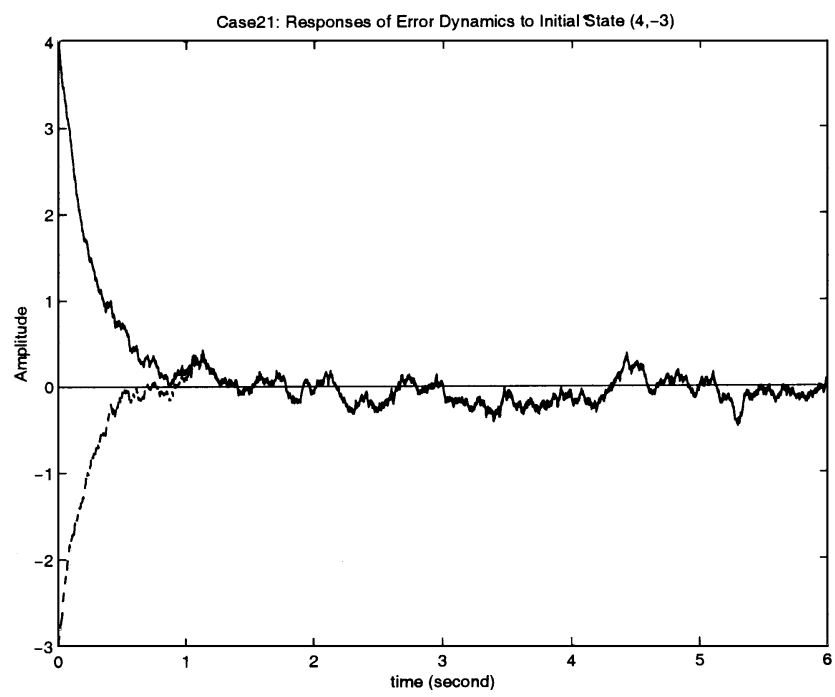

Fig. 3. $e_{1}$ (solid), $e_{2}$ (dash).

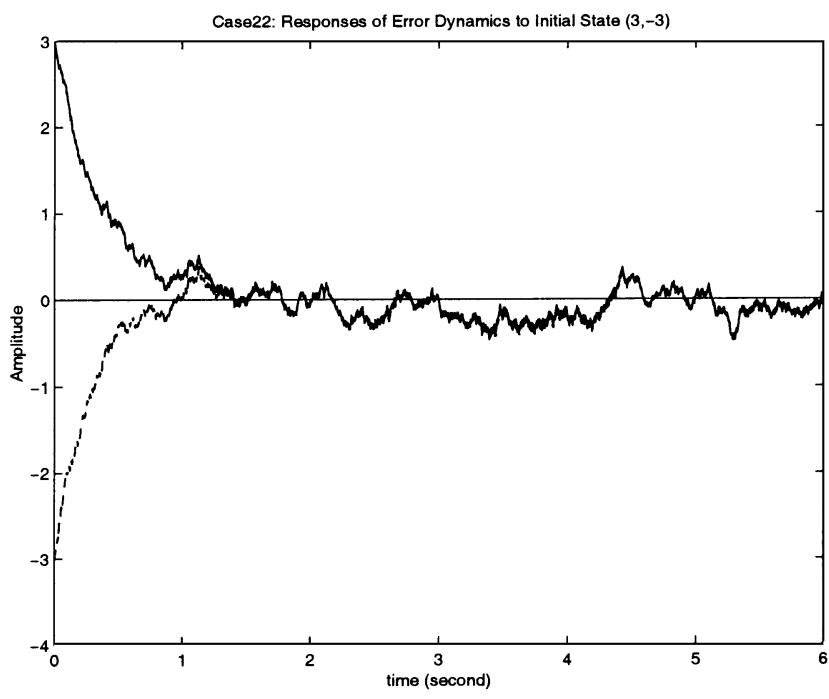

Fig. 4. $e_{1}$ (solid), $e_{2}$ (dash).

\section{CONCLUSION}

In this paper, we have investigated the filter design problem for a class of nonlinear time-delay systems with Markov jumping parameters. Both the filter analysis and design issues have been discussed in detail by means of linear matrix inequalities. We have derived the existence conditions as well as the analytical parameterization of desired filters. The method relies not on the optimization theory but on Lyapunov-type stochastic stability results that can guarantee a mean square exponential rate of convergence for the estimation error. It has been emphasized that the desired exponential filters for this class of nonlinear time-delay systems, when they exist, are usually a large set, and the remaining freedom can be used to meet other expected performance requirements. The results of this paper have been demonstrated by a numerical example.

Finally, we point out that we may generalize our results to more complex systems such as sampled-data systems and stochastic parameter systems, which gives us future research topics.

\section{ACKNOWLEDGMENT}

The authors would like to thank the anonymous referees for their valuable comments that have improved the presentation. Z. Wang is grateful to Professor H. Unbehauen of Ruhr University, Bochum, Germany, and Prof. D. Prätzel-Wolters of the University of Kaiserslautern, Kaiserslautern, Germany for their useful suggestions. He also appreciates several helpful discussions with Dr. D. P. Goodall of Coventry University, Coventry, U.K.

\section{REFERENCES}

[1] S. Boyd, L. El Ghaoui, E. Feron, and V. Balakrishnan, Linear Matrix Inequalities in System and Control Theory, ser. Studies in Applied Mathematics. Philadelphia, PA: SIAM, 1994.

[2] W. H. Fleming and W. M. McEneaney, "A max-plus-based algorithm for a Hamilton-Jacobi-Bellman equation of nonlinear filtering," SIAM J. Contr. Optim., vol. 38, pp. 683-710, 2000.

[3] P. Gahinet, A. Nemirovsky, A. J. Laub, and M. Chilali, LMI Control Toolbox: For Use with Matlab. Natick, MA: The MATH Works Inc., 1995.

[4] A. Gelb, Applied Optimal Estimation. Cambridge, U.K.: Cambridge Univ. Press, 1974.

[5] K. Glover, "All optimal Hankel-norm approximations of linear multivariable systems and their $L^{\infty}$-error bounds," Int. J. Contr., vol. 39, pp. $1115-1193,1984$

[6] Y. Ji and H. J. Chizeck, "Controllability, stabilizability, and continuous-time Markovian jump linear quadratic control," IEEE Trans. Automat. Contr., vol. 35, pp. 777-788, July 1990.

[7] X. Mao, Stochastic Differential Equations and Applications. London, U.K.: Horwood, 1997.

[8] X. Mao, N. Koroleva, and A. Rodkina, "Robust stability of uncertain stochastic differential delay equations," Syst. Contr. Lett., vol. 35, pp. 325-336, 1998.

[9] X. Mao, "Stability of stochastic differential equations with Markovian switching," Stochastic Processes Their Applications, vol. 79, pp. 45-67, 1999.

[10] M. Mariton, Jump Linear Systems in Automatic Control. New York: Marcel Dekker, 1990.

[11] M. S. Mahmoud, N. F. Al-Muthairi, and S. Bingulac, "Robust Kalman filtering for continuous time-lag systems," Syst. Contr. Lett., vol. 38, pp. 309-319, 1999. 
[12] S.-I. Niculescu, E. I. Verriest, L. Dugard, and J. M. Dion et al., "Stability and robust stability of time-delay systems: A guided tour," in Stability and Control of Time-Delay Systems. ser. Lect. Notes Control Inform. Sci., L. Dugard et al., Eds. Berlin, Germany: Springer, 1998, vol. 228, pp. $1-71$.

[13] C. W. Scherer, "Robust generalized $H_{2}$ control for uncertain and LPV systems with general scalings," Proc. IEEE Conf. Decision Contr., pp. 3970-3975, 1996.

[14] P. Shi, E. K. Boukas, and R. K. Agarwal, "Kalman filtering for continuous-time uncertain systems with Markovian jumping parameters," IEEE Trans. Automat. Contr., vol. 44, pp. 1592-1597, Aug. 1999.

[15] T.-J. Tarn and Y. Rasis, "Observers for nonlinear stochastic systems," IEEE Trans. Acoust., Speech, Signal Processing, vol. ASSP-21, pp. 441-448, June 1976.

[16] Z. Wang and K. J. Burnham, "Robust filtering for a class of stochastic uncertain nonlinear time-delay systems via exponential state estimation," IEEE Trans. Signal Processing, vol. 49, pp. 794-804, Apr. 2001.

[17] Z. Wang and F. Yang, "Robust filtering for uncertain linear systems with delayed states and outputs," IEEE Trans. Circuits Syst. I, vol. 49, pp. 125-130, Jan. 2002.

[18] E. Yaz and A. Azemi, "Observer design for discrete and continuous nonlinear stochastic systems," Int. J. Syst. Sci., vol. 24, pp. 2289-2302, 1993.

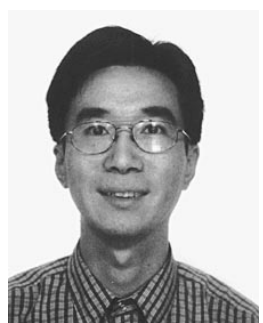

Zidong Wang (M'94) was born in Jiangsu, China, in 1966. He received the B.Sci. degree in mathematics in 1986 from Suzhou University, Suzhou, China, and the M.Sci. degree in applied mathematics in 1990 and the $\mathrm{Ph} . \mathrm{D}$. degree in electrical engineering in 1994, both from Nanjing University of Science and Technology, Nanjing, China.

He was appointed Lecturer at East China Institute of Technology, Nanjing, China, in 1990 and was promoted to Associate Professor at Nanjing University of Science and Technology in 1994. From January 1997 to December 1998, he was an Alexander von Humboldt research fellow with the Automatic Control Laboratory, Ruhr University, Bochum, Germany. From January 1999 to February 2001, he was a Lecturer with the Department of Mathematics, University of Kaiserslautern, Kaiserslautern, Germany. From March 2001 to July 2002, he was a University-funded Senior Research Fellow with the School of Mathematical and Information Sciences, Coventry University, Coventry, U.K. Since August 2002, he has been with the Department of Information Systems and Computing, Brunel University, Uxbridge, U.K., as a Lecturer. His research interests range from data analysis and signal processing to dynamic systems and control, in particular, filtering and control for stochastic systems, uncertain systems, and nonlinear systems. Since 1997, he has published around 40 papers in prestigious international journals as a principal author.

Dr. Wang was awarded the JSPS Research Fellowship in August 1998 from Japan Society for the Promotion of Science and the William Mong Visiting Research Fellowship in November 2002 from the University of Hong Kong. He was a recipient of the Outstanding Science and Technology Development Award from the National Education Committee of China-twice in 1996 and once in 1998 - and the National Science Investigator Award from the National Natural Science Foundation of China in 1995. He is an active reviewer of 16 international journals. In 2000, he was nominated the outstanding reviewer for the journal Automatica, and received Standing Membership of the Technical Committee on Control of the International Association of Science and Technology for Development. From 2001 to 2003, he served as a member of program committee for eight international conferences in the areas of systems theory and computing.

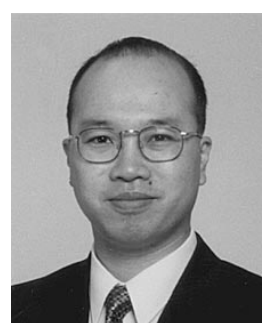

James Lam (SM'99) received a first-class B.Sc. degree in mechanical engineering from the University of Manchester, Manchester, U.K., in 1983. He received the M.Phil. and Ph.D. degrees from the University of Cambridge, Cambridge, U.K., in the area of control engineering in 1985 and 1988, respectively.

He carried out his postdoctoral research with the Australian National University, Canberra, between 1990 and 1992. He has held faculty positions at now the City University of Hong Kong and the University of Melbourne, Parkville, Australia. He is now an Associate Professor with the Department of Mechanical Engineering, the University of Hong Kong, and holds a Concurrent Professorship at the Northeastern University, Shengyang, China, Guest Professorhsip at the Huazhong University of Science and Technology, Wuhan, China, and Consulting Professorship at the South China University of Technology, Guangzhou, China. He also holds a Guest Professorship at Shandong University, Jinan, China. His research interests include model reduction, delay systems, generalized systems, multidimensional systems, robust control and filtering, fault detection, and reliable control. He is an Associate Editor of the Asian Journal of Control and the Interational Journal of Applied Mathematics and Computer Science.

Dr. Lam was awarded the Ashbury Scholarship, the A.H. Gibson Prize, and the H. Wright Baker Prize for his academic performance. He is a Scholar (1984) and Fellow (1990) of the Croucher Foundation, a Chartered Mathematician, a Fellow of the Institute of Mathematics and Its Applications (U.K.), and a Member of the Institution of Electrical Engineers (U.K.).

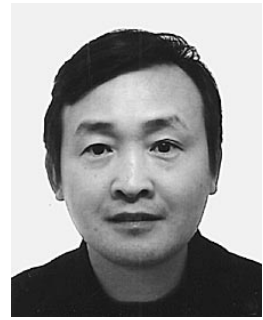

Xiaohui Liu received the B.Eng. degree in computing from Hohai University, Nanjing, China, in 1982 and the Ph.D. degree in computer science from Heriot-Watt University, Edinburgh, U.K., in 1988.

Between October 1987 and October 1989, he was a member of research staff of the Department of Electrical Engineering with both Heriot-Watt University and Durham University, Durham, U.K. $\mathrm{He}$ then joined the Department of Computer Science, Birkbeck College, University of London, London, U.K., for 11 years, first as a Lecturer and, subsequently, a Senior Lecturer. Since October 2000, he has been with the Department of Information Systems and Computing, Brunel University, Uxbridge, U.K., where he is presently Professor of computing and Head of the Intelligent Data Analysis Group, conducting interdisciplinary research involving artificial intelligence, dynamic systems, signal processing, and statistics. He has published more than 80 journal articles/book chapters/conference publications in these areas. He serves on the editorial/review boards of Applied Intelligence Journal, International Journal of Information Technology and Decision Making, and Intelligent Data Analysis Journal.

Dr. Liu founded the biennial Intelligent Data Analysis conference series in 1995 and has been on the technical program committees of various international conferences. He has been a principal investigator of numerous research projects funded by research councils, charities and industries. In 2002, he gave a keynote address at the International Conference of the Royal Statistical Society in the U.K. 